\title{
Epstein-Barr virus renders the infected natural killer cell line, NKL resistant to doxorubicin-induced apoptosis
}

\author{
Y Isobe', K Sugimoto*,', I Matsuura', K Takada ${ }^{2}$ and K Oshimi' \\ 'Department of Hematology, Juntendo University School of Medicine, 2-I I Hongo, Bunkyo-ku, Tokyo I I 3-842 I, Japan; '2 Department of Tumor Virology, \\ Institute for Genetic Medicine, Hokkaido University, NI 5 W7 Kita-ku, Sapporo 060-8638, Japan
}

\begin{abstract}
We established two Epstein-Barr virus (EBV)-infected NKL sublines, which acquired stress resistant phenotype against DNA damage and starvation compared with EBV-negative NKL. EBV-rendered doxorubicin resistance at least partially through NF- $\kappa \mathrm{B}$ activation and the resultant sustenance of antiapoptotic proteins including $B C l-X_{L}$ and FLIPL/S.

British Journal of Cancer (2008) 99, I8I6- |822. doi:I0.1038/sj.bjc.6604764 www.bjcancer.com

Published online 4 November 2008

(c) 2008 Cancer Research UK
\end{abstract}

Keywords: natural killer-cell malignancies; Epstein-Barr virus; apoptosis; doxorubicin; nuclear factor- $\kappa$ B

Natural killer (NK)-cell malignancies including nasal-type NK-cell lymphoma and aggressive NK-cell leukaemia have been recently recognised as new disease entities, which frequently arise in east Asia and central America and present poor prognosis (Oshimi, 2007). These diseases are closely associated with Epstein-Barr virus (EBV) infection (Kawa-Ha et al, 1989; Kaneko et al, 1995; Oshimi, 2007). EBV genome was detected in approximately $98 \%$ of nasal-type NK-cell lymphoma cases and $83 \%$ of aggressive NK-cell leukaemia cases in Japan (Oshimi et al, 2002). The presence of monoclonal EBV in neoplastic NK cells indicates that EBV infection occurs prior to the clonal expansion.

EBV immortalises B cells in vitro. In this situation, EBV expresses several latent gene products including six EBV-determined nuclear antigens, three latent membrane proteins, and two EBV-encoded RNAs (EBERs) (Young and Rickinson, 2004). Although these EBVderived molecules function as transactivators and signal transducers in the infected cells, most of these molecules except for EBNA1, EBERs and occasional LMP1 are not expressed in nasal-type NK-cell lymphoma (Xu et al, 2001). EBNA1, EBERs and LMP1 also contribute to cellular transformation by affecting cellular gene expression and protein stability (Nambo and Takada, 2002; Masucci, 2004; Young and Rickinson, 2004; Samanta et al, 2006). Until now, it has not sufficiently elucidated the pathogenetic role of EBV in NK-cell malignancies. Recently, we have shown that EBV directly infects human NK cells in vitro (Isobe et al, 2004). In vitro EBV infection of NK cells enabled us to establish two EBV-carrying NK-cell sublines and to evaluate their phenotypic changes.

\section{MATERIALS AND METHODS}

\section{Establishment of EBV-infected NKL sublines}

Cell-culture condition and infection procedure were described earlier (Isobe et al, 2004). We used EBV-negative NK-cell leukaemia

*Correspondence: Dr K Sugimoto; E-mail: ksugimot@juntendo.ac.jp Revised 3 October 2008; accepted 6 October 2008; published online 4 November 2008 cell line, NKL as an EBV target cell (Robertson et al, 1996). NKL was maintained in Iscove's modified Dulbecco's medium (Invitrogen, Carlsbad, CA, USA) containing $100 \mathrm{U} \mathrm{ml}^{-1}$ human interleukin (IL)-2 (a gift from Shionogi, Osaka, Japan). We previously confirmed that this cell line had no mutations in exon 5-to-8 of TP53 gene (Sakajiri et al, 2001). Akata (EBV-positive) and BJAB (EBV-negative) were used as an EBV-producing cell and an EBV target control, respectively. Akata is infected with recombinant EBV strain containing neomycin-resistant gene (Yoshiyama et al, 1995). After selection with medium containing $700 \mu \mathrm{g} \mathrm{ml}^{-1}$ of G418 (Invitrogen), EBV-infected clones were maintained for more than four years using G418-free medium. BJAB was obtained from Fujisaki Cell Center, Hayashibara biochemical laboratories Inc. (Okayama, Japan).

\section{Southern blot analysis, western blot analysis, and flow cytometry}

Southern blot analysis was performed using EBV $1.9 \mathrm{~kb}$ XhoI probe and detected with CSPD detection system (Roche Diagnostics, Basel, Switzerland). Western blot analysis was performed using the following antibodies: anti-EBNA1 (Advanced Biotechnologies, Columbia, MD, USA); anti-EBNA2; anti-LMP1; anti-ZEBRA, (Dako, Glostrup, Denmark); anti-early antigen diffuse (EA-D), (Chemicon, Temecula, CA, USA); anti-Bcl-2 (Dako); anti-Bcl-X $\mathrm{X}_{\mathrm{L}}$; anti-Mcl-1 (Santa Cruz Biotechnology, Santa Cruz, CA, USA); anti-Bax (Cell Signaling Technology, Danvers, MA, USA); anti-FLIP (Alexis, Lausen, Switzerland); anti-FLIP $\gamma / \delta$ (Sigma-Aldrich, Stockholm, Sweden); anti-Hsp 90 (Nventa, San Diego, CA, USA); anti-p53 (Santa Cruz); anti-phosphorylated p53 (Cell Signaling Technology); and anti- $\beta$ actin antibody (Sigma-Aldrich). Antibody signals were enhanced and detected with ECL (Amersham International plc, Buckinghamshire) or Western Blue (Promega, Madison, WI, USA). Cell-surface P-glycoprotein (P-gp) was analysed by flow cytometry using anti-P-gp antibody, MRK16 (Kyowa Medex, Tokyo, Japan).

\section{Cell viability and apoptosis assay}

Viable and dead cell numbers were determined by trypan-blue dye exclusion. Each cell suspension $\left(4 \times 10^{5} \mathrm{ml}^{-1}\right)$ was treated with 
A

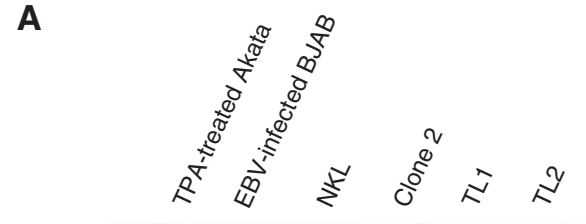
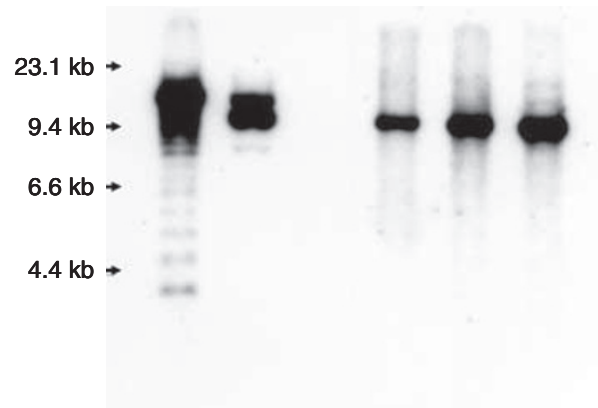

$2.3 \mathrm{~kb}$ -

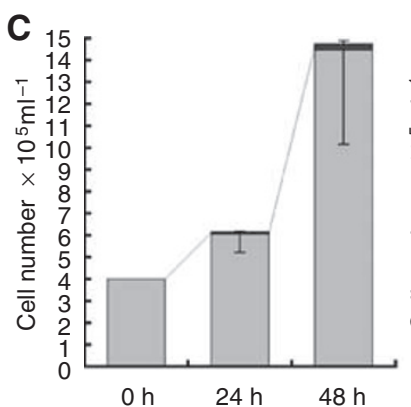

NKL
B

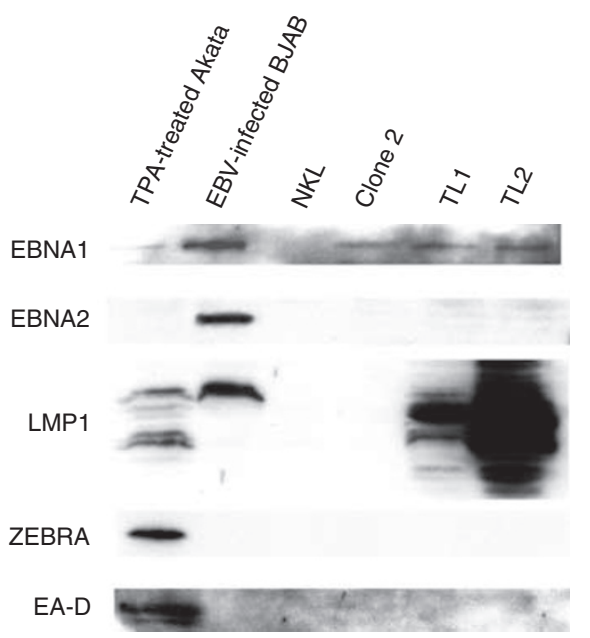

E

NKL
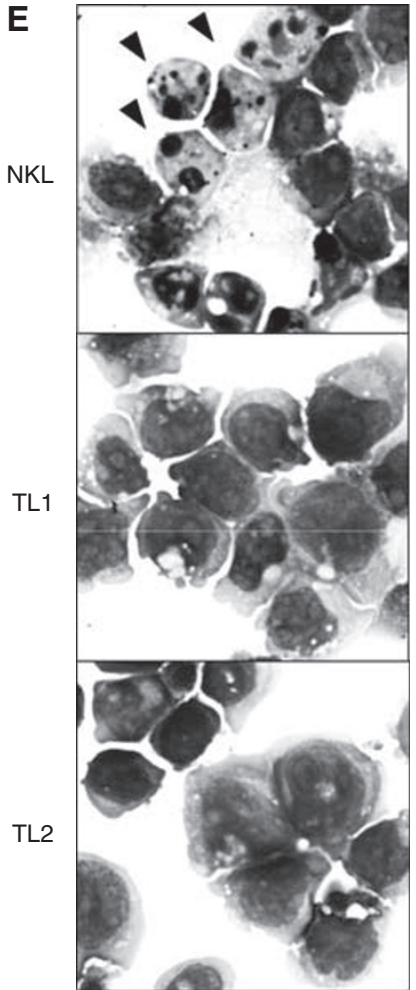

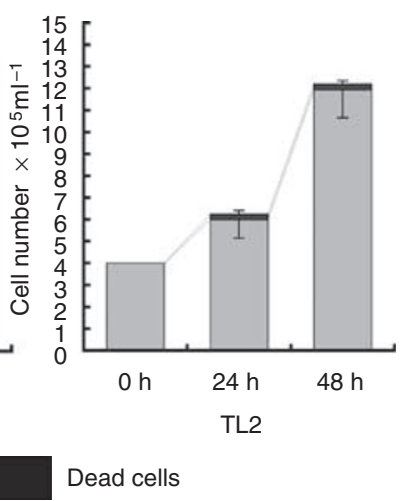

Dead cells

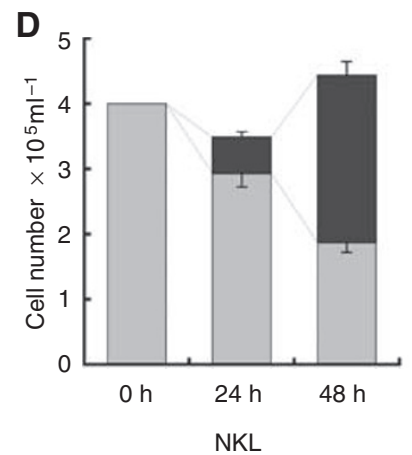

NKL
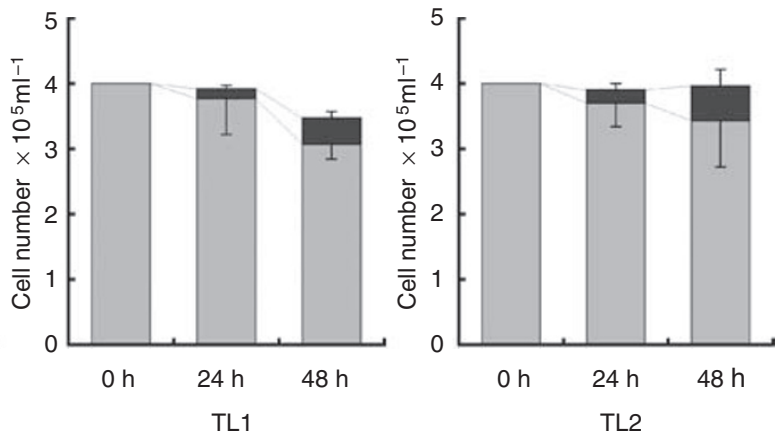

Alive cells

Dead cells

Figure I (A) NKL and BJAB were infected with Akata-derived EBV. Southern blot analysis detected monoclonal EBV genome in EBV-infected NKL cells. The blotting signal for clone 2 (lane 4) is weaker than those of established EBV-infected NKL sublines named TLI (lane 5) and TL2 (lane 6), suggesting that clone 2 should contain less copy number of EBV. EBV-infected BJAB appears to consist of two clones (lane 2). (B) TLI (lane 5) and TL2 (lane 6) express EBNAI and LMPI, but lack EBNA2 and lytic marker proteins, ZEBRA and EA-D. TPA-treated Akata (lane I) and EBV-infected BJAB (lane 2) are positive controls of lytic and latent phases, respectively. (C) Time courses of cell count for NKL, TLI, and TL2 at steady state. TLI and TL2 showed no growth advantage compared with NKL. Alive (trypan-blue negative)- and dead (trypan-blue positive)-cell counts are shown in gray- and black-bars, respectively. (D) Time courses of cell count for NKL, TLI, and TL2 after treatment with $150 \mathrm{nM}$ of doxorubicin (DXR). After $48 \mathrm{~h}$, approximately $60 \%$ of NKL cells were dead, whereas above $85 \%$ of TLI and TL2 cells survived. (E) Change in cell morphology after $48 \mathrm{~h}$ of treatment with DXR in three lines (Wright-Giemsa stain $\times 1000$ ). Although both TLI and TL2 cells had no apparent change in their cell morphology, about half of NKL cells underwent apoptosis (arrowheads). (F) A cell viability assay using 3-(4,5-dimethylthiazol-2-yl)-2,5-diphenyltetrazolium bromide after $48 \mathrm{~h}$ of treatment with 4-hydroxycyclophosphamide, DXR, vincristine, and BMS-34554I, TLI and TL2 showed resistance to DXR and VCR treatment compared with NKL. (G) Flow cytometric terminal deoxynucleotidyl transferase-mediated dUTP nick end-labeling (TUNEL) assay after treatment with I50 nM of DXR or serum depletion (from I0 to $0.1 \%$ of fetal bovine serum). Although about half of NKL cells underwent apoptosis after each treatment, essentially no apoptotic populations were detected in TLI and TL2. Open arrowheads show TUNEL-positive populations. $(\mathbf{H}) 7$-amino actinomycin D rejection and annexin V-binding assay after treatment with DXR or serum depletion. About half of NKL cells became positive for annexin $V$ after each treatment. In contrast, approximately $90 \%$ of TLI and TL2 cells were negative for annexin $\vee$ after each treatment. 
$\mathbf{F}$
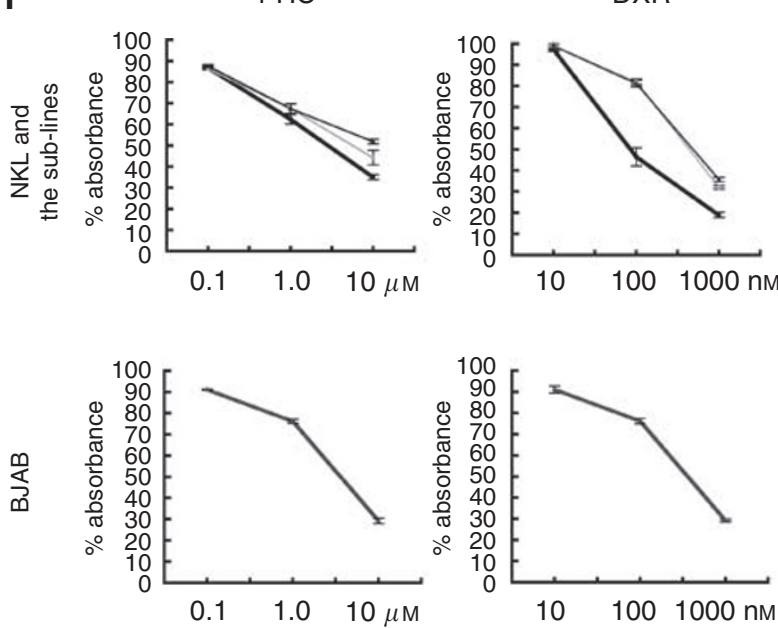

G

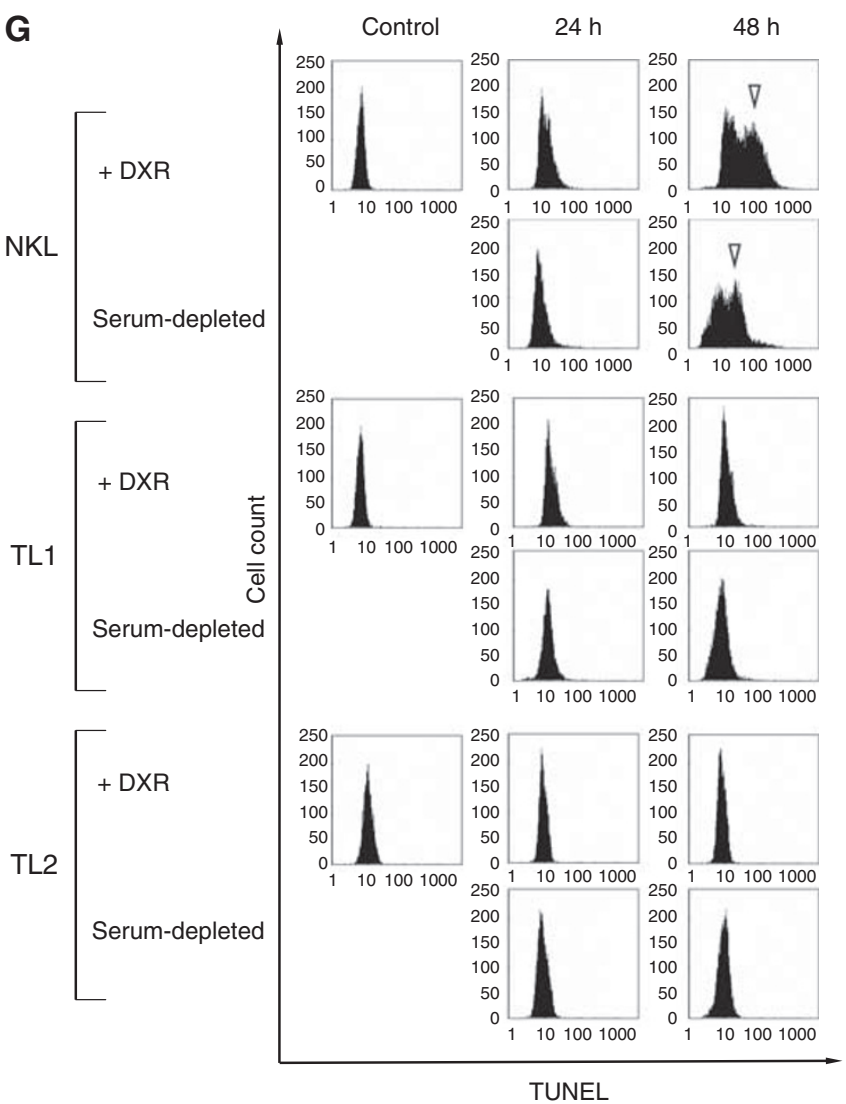

Figure I Continued.

4-hydroxycyclophosphamide (HC) (a gift from Shionogi), doxorubicin (DXR), vincristine (VCR) (Sigma-Aldrich), and I- $\kappa$ B kinase inhibitor BMS-345541 (Merck KGaA, Darmstadt, Germany). We assessed the cell viability using 3-(4,5-dimethylthiazol-2-yl)-2,5diphenyltetrazolium bromide (MTT) assay (Promega). The viability was calculated as percent absorbance of formazan products, that is, (OD570DXR-treated/OD570control) $\times 100 \%$. We also evaluated the cell death process by FITC-conjugated annexin V-binding and 7-amino actinomycin D (AAD)-rejection assays (Beckman Coulter, Fullerton, CA, USA). DNA nick ends in apoptotic cells were labelled with fluorescein isothiocyanate
VCR
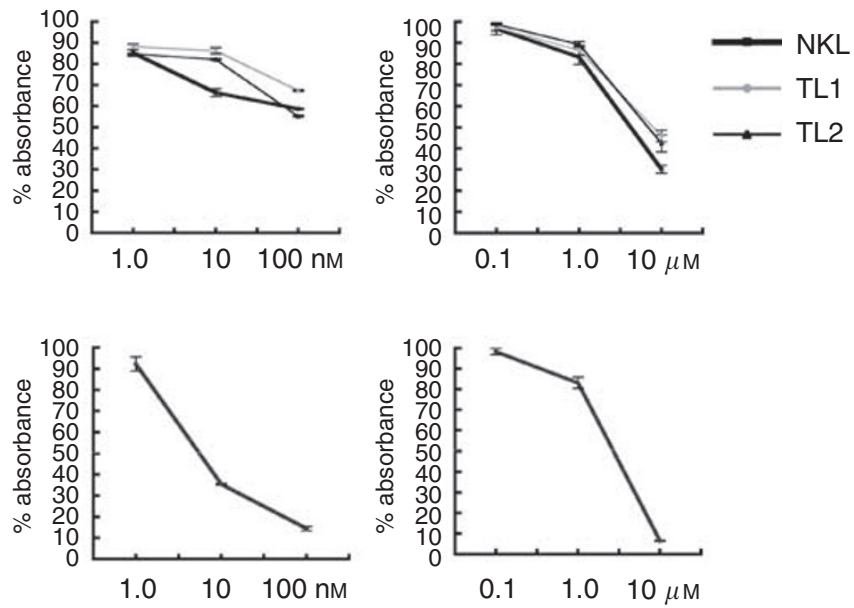

H
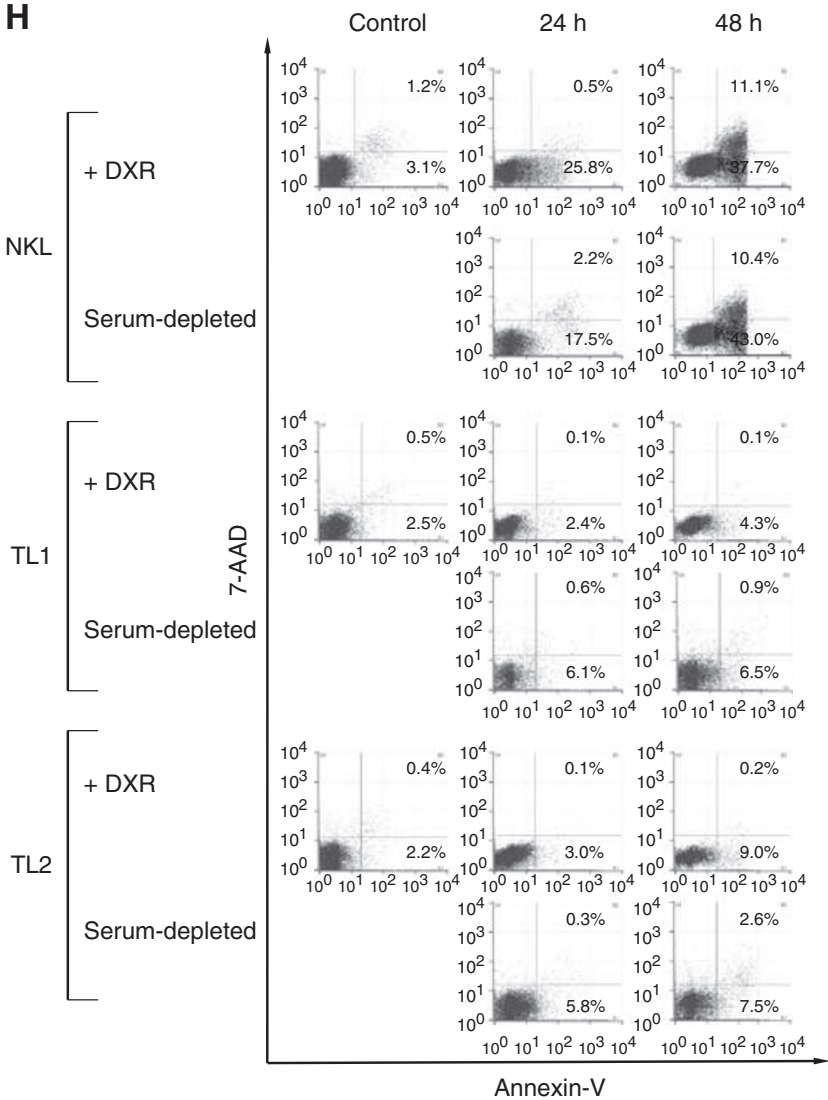

(FITC)-conjugated dUTP using Mebstain Apoptosis Kit (Beckman Coulter) and analysed by flow cytometry.

\section{Nuclear factor- $\kappa \mathrm{B}$ activity assay}

DNA binding activity of nuclear factor (NF) $-\kappa \mathrm{B}$ was evaluated using an enzyme-linked immunosorbent assay method. Nuclear extract $(10 \mu \mathrm{g})$ was isolated from DXR-treated and untreated cells and assayed using NF- $\kappa$ B p65 Transcription Factor assay Kit (Chemicon). Relative absorbance was calculated as follows: OD450/OD650 - OD450control/OD650control. 


\section{RESULTS}

\section{Establishment of EBV-carrying NKL sublines}

We initially obtained three G418-resistant NKL subclones containing monoclonal EBV genome (Figure 1A). During the 4 years of incubation, clone 2 stopped proliferation, and we finally established two EBV-infected sublines named TL1 and TL2. Western blot analysis showed expression of EBNA1 in all EBV-infected subclones (Figure 1B). EBNA2 and lytic marker proteins, ZEBRA and EA-D were absent in these clones. In addition, LMP1 was detected in TL1 and TL2 but not in clone 2. LMP1 in TL1 and TL2 showed smaller sizes than that in EBV-infected BJAB (Figure 1B). These truncated forms resemble lytic LMP1 detected in TPA-treated Akata (Figure 1B). Because lytic marker proteins were absent in both sublines, detection of the truncated forms may represent rapid turnover of LMP1 in TL1 and TL2.
EBV infection of NKL showed no growth advantage but rendered the infected cells resistant to doxorubicininduced apoptosis

NKL and EBV-infected TL1 and TL2 showed no difference in cell growth at a steady state (Figure 1C). After treatment with $150 \mathrm{~nm}$ of DXR, both sublines remained more viable than NKL, that is, although approximately $60 \%$ of NKL cells became positive for trypan-blue dye exclusion assay after $48 \mathrm{~h}$, less than $14 \%$ of TL1 and TL2 cells were dead (Figure 1D). Indeed, about half of NKL cells underwent apoptosis after the DXR treatment (Figure 1E). We also evaluated possible difference of drug sensitivity among NKL, TL1 and TL2 using 4-HC, DXR, VCR, and I- $\kappa$ B kinase inhibitor BMS-345541. After $48 \mathrm{~h}$ of treatment, MTT assay showed that TL1 and TL2 were more resistant to DXR than NKL (Figure 1F). Although NKL itself was rather resistant to VCR compared with $\mathrm{BJAB}$, the sublines were a little more refractory to the agent. The

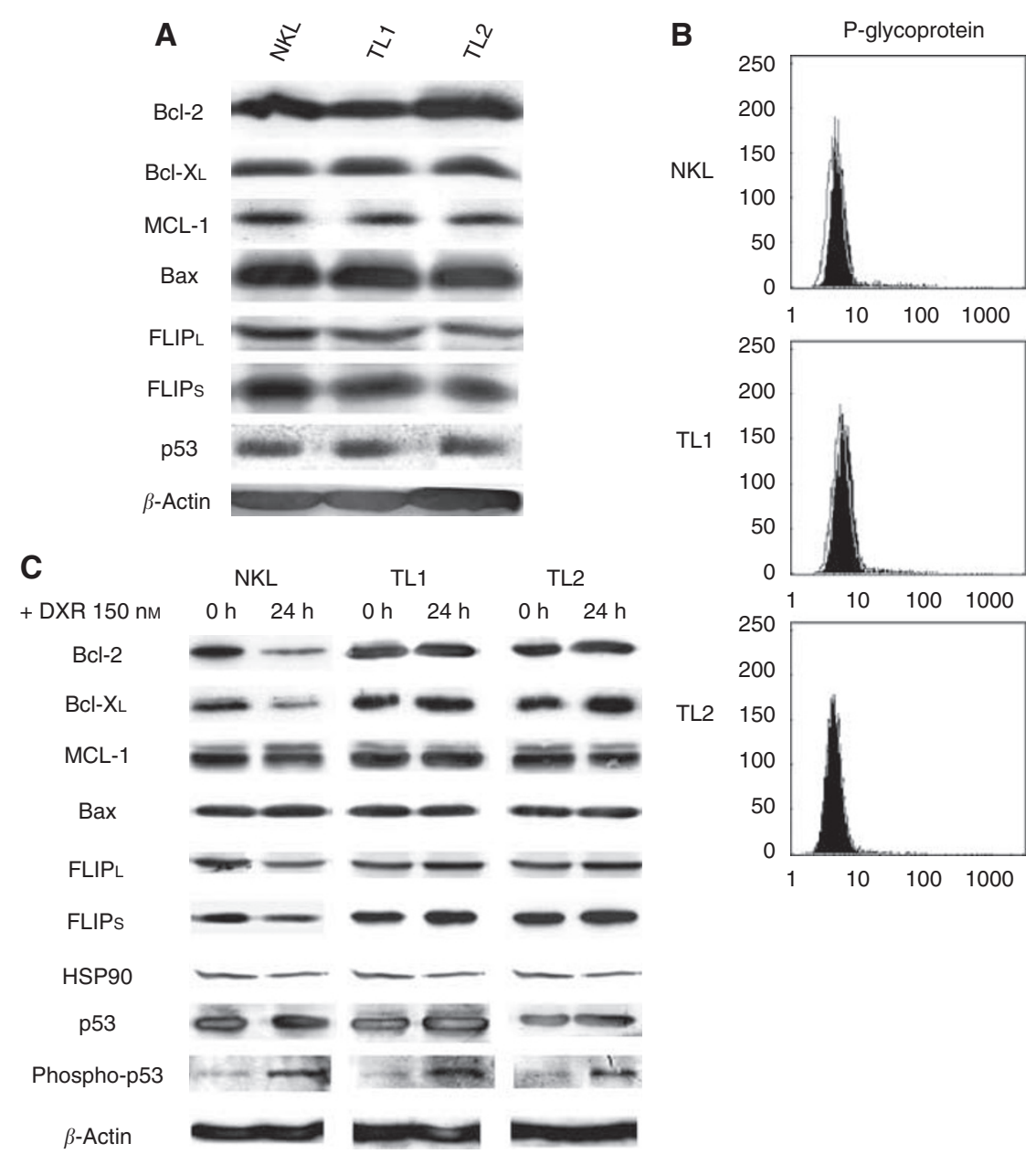

Figure 2 (A) Western blot analysis showed essentially the same expression levels of Bcl-2, Bcl-X, Mcl- I, Bax, FLIP $L /$, and p53 among NKL, TLI and TL2. (B) Cell-surface P-glycoprotein was absent not only in NKL but also TLI and TL2 by flow cytometry. Open histograms show negative control with isotypematched control antibody. (C) Alterations of antiapoptotic protein levels in NKL and two sublines after doxorubicin (DXR) treatment. Although NKL declined expression levels of $\mathrm{BCl}-2, \mathrm{Bcl}-\mathrm{X}_{\mathrm{L}}$, and FLIP $\mathrm{Ss}$ after $24 \mathrm{~h}$ of DXR treatment (left column), TLI (middle column) and TL2 (right column) kept to express these proteins, and showed rather increased expression levels of $B C l-X_{L}$ and FLIP $L / s$. (D) NF- $\kappa B$ (p65 subunit) binding activity after DXR treatment. Although the binding activity in NKL remained to be its basal level at $24 \mathrm{~h}$, those in TLI and TL2 increased approximately five times after $12 \mathrm{~h}$ and further boosted at $24 \mathrm{~h}$. In contrast, NF- $\kappa$ B inhibitor BMS-34554I almost completely suppressed DXR-induced NF- $\kappa$ B activation in all three lines. Each experiment was performed in triplicate. Each error bar represents s.d. (E) Alterations of antiapoptotic protein levels in NKL and two sublines after treatment with both DXR and BMS-34554I. In the presence of BMS-34554I, treatment with DXR clearly decreased expression levels of BCl-X $X_{L}$ and FLIPLS even in TLI and TL2. Expression level of Bcl-2 was decreased only in NKL. (F) 7-amino actinomycin D rejection and annexin $\mathrm{V}$-binding assay after treatment with DMSO (control), BMS-34554I, DXR, and both BMS-34554I and DXR. Although approximately 5-7\% of apoptotic populations were detected in TLI and TL2 after treatment with $3 \mu \mathrm{M}$ of BMS-34554I and I50 nM of DXR, respectively, those mixed treatments induced apoptosis in approximately $40 \%$ of populations in TLI and TL2. 

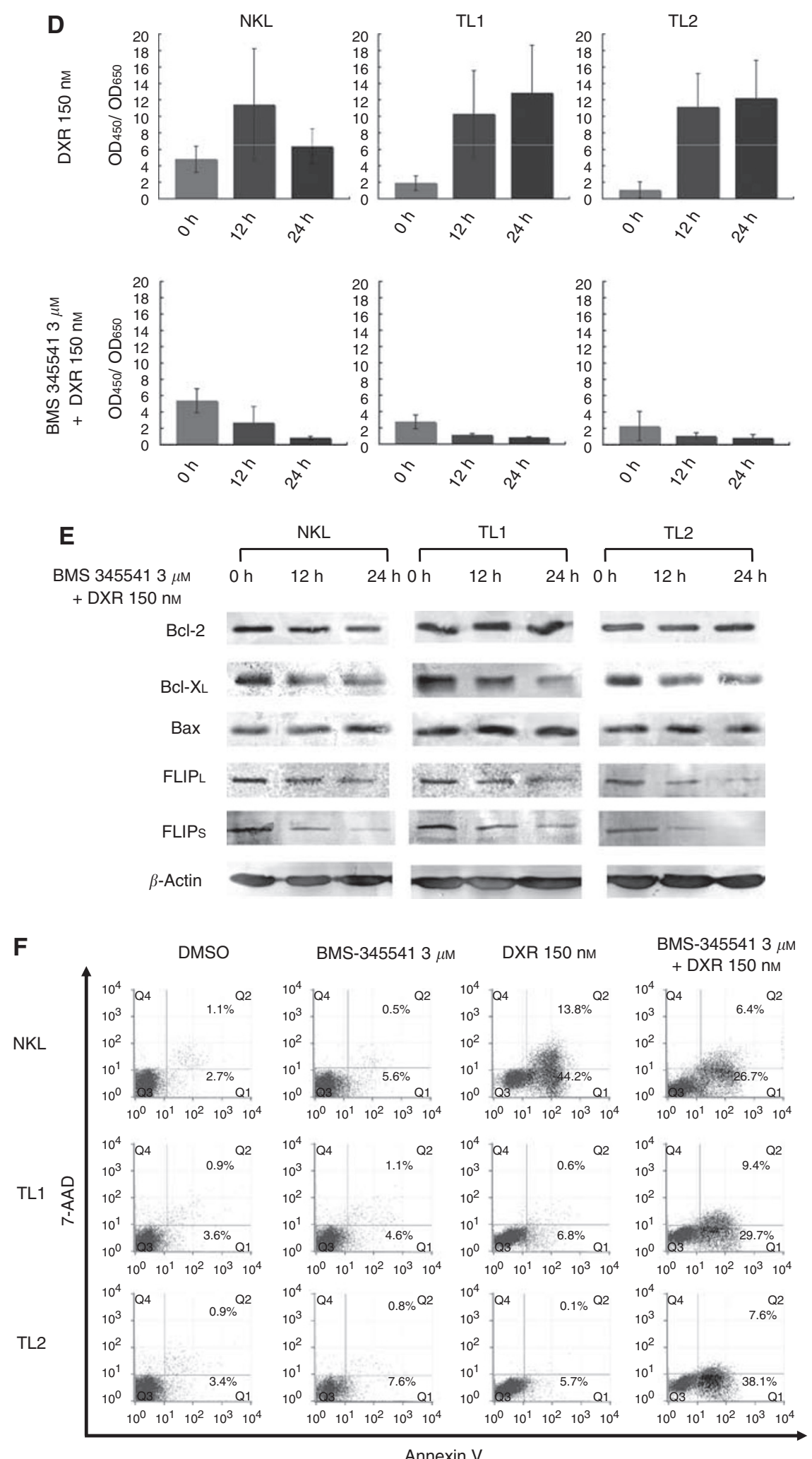

Figure 2 Continued.

effect of 4-HC or BMS-345541 on cell viability was almost equal between NKL and its sublines. Flow cytometric terminal deoxynucleotidyl transferase-mediated dUTP nick end-labelling assay showed that treatment with $150 \mathrm{~nm}$ of DXR or serum depletion $(10 \%$ to $0.1 \%)$ for $48 \mathrm{~h}$ induced apoptosis in about half of NKL cells but in only few TL1 and TL2 cells (Figure 1G). Annexin V-binding and 7-AAD-rejection assays confirmed the loss of susceptibility to DXR and serum depletion in TL-1 and TL-2 (Figure 1H). These results showed that EBV rendered the infected NKL cells resistant to various cell stresses like DNA damage and starvation. 
We next evaluated possible alterations of antiapoptotic proteins after EBV infection of NKL cells. Western blot analysis showed essentially the same levels of Bcl-2, Bcl- $\mathrm{X}_{\mathrm{L}}, \mathrm{Mcl}-1, \mathrm{Bax}, \mathrm{p} 53$ and FLIP $_{\mathrm{L} / \mathrm{S}}$ among NKL, TL1 and TL2 (Figure 2A). Expression of MDR1-encoded P-gp was absent in all these lines, which confirmed that the resistance to DXR should be unrelated to drug pumping (Figure 2B). Although no apparent difference was detected at the steady state, treatment with $150 \mathrm{~nm}$ of DXR for $24 \mathrm{~h}$ reduced expression levels of Bcl-2, Bcl- $\mathrm{X}_{\mathrm{L}}$, and $\mathrm{FLIP}_{\mathrm{L} / \mathrm{S}}$ specifically in NKL (Figure 2C). The amounts of these proteins were constant or rather increased in TL1 and TL2 after the same treatment. Protein levels of Mcl-1 and Bax were unchanged in all three lines (Figure 2C). Increased levels of p53 phosphorylation at serine-15 after DXR treatment indicated preservation of normal p53 activity in all three lines (Figure 2C). Because nuclear factor (NF) $\kappa \mathrm{B}$ is reported to affect expression levels of $\mathrm{Bcl}-\mathrm{X}_{\mathrm{L}}$ and $\mathrm{FLIP}_{\mathrm{L} / \mathrm{S}}$ (Karin, 2006), we examined possible involvement of NF- $\kappa \mathrm{B}$ in the EBV-mediated resistance to DXR. In spite of a transient increase at $12 \mathrm{~h}$, target sequences-binding activity of NF- $\kappa$ B p65 clearly regressed after $24 \mathrm{~h}$ of DXR treatment in NKL (Figure 2D). In contrast, although the basal activities in TL1 and TL2 were rather suppressed, they increased approximately five times as high as their basal levels after $12 \mathrm{~h}$ of DXR treatment, and were further boosted at $24 \mathrm{~h}$ (Figure 2D). However, NF- $\kappa$ B inhibitor BMS-345541 almost completely suppressed DXR-induced NF- $\kappa$ B activation in all three lines. In the presence of this inhibitor, treatment with DXR clearly decreased expression levels of $\mathrm{Bcl}-\mathrm{X}_{\mathrm{L}}$ and $\mathrm{FLIP}_{\mathrm{L} / \mathrm{S}}$ and induced massive apoptosis even in TL1 and TL2 (Figure 2E and F). It should be noted that BMS-345541 rather decreased the apoptotic cell percentage from approximately $60 \%$ to around $35 \%$ in DXRtreated NKL. These experiments were repeated at least three times with essentially the same results.

\section{DISCUSSION}

In this study, we established TL1 and TL2, which contain monoclonal EBV genome and showed type II latency. Their manner of infection corresponds to the clinical setting of NK-cell malignancies (Xu et al, 2001). The intense signals in Southern blot analysis suggested that TL1 and TL2 should contain greater copy number of EBV genome than clone 2 (Figure 1A). Relatively high copy number of EBV may contribute to successful maintenance of TL1 and TL2 over 4 years. We previously detected truncated forms of LMP1 at a later phase of latent infection (Isobe et al, 2004). Although full-length LMP1 induces NF- $\kappa$ B activation, its truncated form is reported to inhibit this effect (Erickson and Martin, 2000). The canonical function of LMP1 seems to be limited and might not fully contribute to establish the latent infection in both NKL sublines. These truncated forms might explain the relatively low basal NF- $\kappa$ B activity in TL1 and TL2 compared with NKL. Of course, latent EBV infection may exert a supportive role for the NF- $\kappa \mathrm{B}$ pathway and might lessen its activity without altering basal cell growth activity in both sublines.

At a steady state, TL1 and TL2 had no growth advantage and showed essentially the same expression levels of several antiapoptotic proteins as NKL. However, these EBV-infected sublines were apparently more resistant to DXR and serum depletion than NKL. The results indicate that EBV infection conferred resistance to various cell stresses like DNA damage and starvation in NKL. Expression levels of Bcl-2, Bcl- $\mathrm{X}_{\mathrm{L}}$ and $\mathrm{FLIP}_{\mathrm{L} / \mathrm{s}}$ decreased in NKL but not in two sublines after DXR treatment. In the presence of NF- $\kappa$ B inhibitor BMS-345541, both NKL and the EBV-infected sublines were almost equally sensitive to DXR and failed to maintain the expression levels of Bcl- $\mathrm{X}_{\mathrm{L}}$ and $\mathrm{FLIP}_{\mathrm{L} / \mathrm{S}}$ after DXR treatment. The results are completely in line with the previous report that constitutively activated NF- $\kappa \mathrm{B}$ has shown to maintain high expression levels of $\mathrm{Bcl}-\mathrm{X}_{\mathrm{L}}$ and $\mathrm{FLIP}_{\mathrm{L} / \mathrm{S}}$ and confer resistance to some anticancer drugs (Wang et al, 1999; Karin, 2006). EBVassociated gene products like EBNA1 and LMP1 were reported to modify the ubiquitin - proteasome activity and affect proteolysis of various cellular proteins including NF- $\kappa \mathrm{B}$ (Masucci, 2004). In addition, EBERs appear to be involved in modulation of cellular response and were reported to activate NF- $\kappa \mathrm{B}$-signalling pathway (Nambo and Takada, 2002; Samanta et al, 2006). These observations strongly argue that the presence of EBV must render NKL more resistant to lethal stress like DNA damage at least partially through the NF- $\kappa$ B pathway.

During a series of experiments aimed to show the contribution of NF- $\kappa \mathrm{B}$ activity in stress resistance of TL1 and TL2, we found that BMS-345541 reproducibly attenuated DXR-induced apoptosis in NKL cells. Atypical activators of NF- $\kappa \mathrm{B}$ including DXR have been shown to convert p65 from a transcriptional activator into a transcriptional repressor of antiapoptotic genes including $\mathrm{Bcl}-\mathrm{X}_{\mathrm{L}}$ and $\mathrm{FLIP}_{\mathrm{L} / \mathrm{S}}$ (Campbell et al, 2004; Dutta et al, 2006). We believe that DXR may induce apoptosis in NKL at least partially through this atypical activation of NF- $\kappa \mathrm{B}$. Prolonged activation of NF- $\kappa \mathrm{B}$ during DXR treatment maintained high expression levels of $\mathrm{Bcl}-\mathrm{X}_{\mathrm{L}}$ and FLIP $_{\mathrm{L} / \mathrm{S}}$ in the EBV-infected sublines. Therefore, we suppose that in the presence of some EBV-associated gene products, even DXR treatment may preferentially elicit classical NF- $\kappa$ B activity with an antiapoptotic feature rather than inducing the proapoptotic situation and thus render the cell resistant to apoptosis. Abrogation of both positive and negative impacts of NF- $\kappa$ B on apoptosis by BMS-345541 should bring about essentially the same behaviour of NKL, TL1 and TL2 toward the DXR treatment.

In conclusion, we report here in vitro establishment of two EBV-infected NKL sublines, TL1 and TL2. Although the infected sublines had no growth advantage compared with NKL, they showed a resistant phenotype to stress-induced apoptosis at least partially through the NF- $\kappa \mathrm{B}$ activation and the resultant sustenance of $\mathrm{Bcl}-\mathrm{X}_{\mathrm{L}}$ and $\mathrm{FLIP}_{\mathrm{L} / \mathrm{s}}$. We believe that this model suggests the contribution of EBV in NK-cell tumorigenesis.

\section{ACKNOWLEDGEMENTS}

This work was supported by grant-in-aid for Scientific Research from the Japanese Ministry of Education, Culture, Sports, Science, and Technology. We greatly thank Dr Michael J Robertson (Indiana University, Indianapolis, IN, USA) and Dr Yuan Yuan $\mathrm{Xu}$ for providing NKL cells and technical assistance, respectively.

\section{REFERENCES}

Campbell KJ, Rocha S, Perkins ND (2004) Active repression of antiapoptotic gene expression by Rel A (p65) NF- $\kappa$ B. Mol Cell 13: $853-865$

Dutta J, Fan Y, Gupta N, Fan G, Gélinas C (2006) Current insights into the regulation of programmed cell death by NF- $\kappa$ B. Oncogene 25: $6800-6816$

Erickson KD, Martin JM (2000) The late lytic LMP-1 protein of EpsteinBarr virus can negatively regulate LMP-1 signaling. J Virol 74: 1057- 1060
Isobe Y, Sugimoto K, Yang L, Tamayose K, Egashira M, Kaneko T, Takada K, Oshimi K (2004) Epstein-Barr virus infection of human natural killer cell lines and peripheral blood natural killer cells. Cancer Res 64: 2167-2174 Karin M (2006) Nuclear factor- $\kappa$ B in cancer development and progression. Nature 441: $431-436$

Kaneko T, Fukuda J, Yoshihara T, Zheng H, Mori S, Mizoguchi H, Oshimi K (1995) Nasal natural killer (NK) cell lymphoma: report of a case with 
activated NK cells containing Epstein-Barr virus and expressing CD21 antigen, and comparative studies of their phenotype and cytotoxicity with normal NK cells. Br J Haematol 91: 355-361

Kawa-Ha K, Ishihara S, Ninomiya T, Yumura-Yagi K, Hara J, Murayama F, Tawa A, Hirai K (1989) CD3-negative lymphoproliferative disease of granular lymphocytes containing Epstein-Barr viral DNA. J Clin Invest 84: 51 - 55

Masucci MG (2004) Epstein-Barr virus oncogenesis and the ubiquitinproteasome system. Oncogene 23: 2107-2115

Nambo A, Takada K (2002) The role of Epstein-Barr virus-encoded small RNAs (EBERs) in oncogenesis. Rev Med Virol 12: 321-326

Oshimi K, Kawa K, Nakamura S, Suzuki R, Suzumiya J, Yamaguchi M, Kameoka J, Tagawa S, Imamura N, Ohshima K, Kojya S, Iwatsuki K, Tokura Y, Sato E, Sugimori H (2002) NK-cell neoplasms in Japan. Hematology 10: $250-256$

Oshimi K (2007) Progress in understanding and managing NK-cell malignancies. Br J Haematol 139: 532-544

Robertson MJ, Cochran KJ, Cameron C, Le JM, Tantravahi R, Ritz J (1996) Characterization of a cell line, NKL, derived from an aggressive human natural killer cell leukemia. Exp Hematol 24: 406-415
Sakajiri S, Kawamata N, Egashira M, Mori K, Oshimi K (2001) Molecular analysis of tumor suppressor genes, Rb, p53, p16INK4A, p15INK4B and p14ARF in natural killer cell neoplasms. Jpn J Cancer Res 92: $1048-1056$

Samanta M, Iwakiri D, Kanda T, Imaizumi T, Takada K (2006) EB virusencoded RNAs are recognized by RIG-I and activate signaling to induce type I IFN. EMBO J 25: 4207-4214

Wang CY, Cusack Jr JC, Liu R, Baldwin Jr AS (1999) Control of inducible chemoresistance: enhanced anti-tumor therapy through increased apoptosis by inhibition of NF- $\kappa$ B. Nat Med 5: 412-417

Xu ZG, Iwatsuki K, Oyama N, Ohtsuka M, Satoh M, Kikuchi S, Akiba H, Kaneko F (2001) The latency pattern of Epstein-Barr virus infection and viral IL-10 expression in cutaneous natural killer/T-cell lymphomas. Br J Cancer 84: 920 - 925

Yoshiyama H, Shimizu N, Takada K (1995) Persistent Epstein-Barr virus infection in a human T-cell line: unique program of latent virus expression. $E M B O J$ 14: $3706-3711$

Young LS, Rickinson AB (2004) Epstein-Barr virus: 40 years on. Nat Rev Cancer 4: $757-768$ 